|| ISSN(online): 2589-8698 || ISSN(print): 2589-868X || International Journal of Medical and Biomedical Studies

Available Online at www.ijmbs.info

PubMed (National Library of Medicine ID: 101738825)

Index Copernicus Value 2018: 75.71

Volume 3, Issue 4; April: 2019; Page No. 139-144

\title{
A STUDY TO EVALUATE THE REACTION TIME IN TENSION TYPE HEADACHE.
}

Dr. Mukta P Bidikar ${ }^{1}$, Dr. Gayatri Jagtap ${ }^{2}$, Dr. Rahul Chakor ${ }^{3}$

${ }^{1}$ Assistant Professor, Department of Physiology, HBT Medical College \& Dr. R N Cooper Hospital, Mumbai

${ }^{2}$ Senior Medical Officer, Department of Physiology, Topiwala National Medical College \& BYL Nair Charitable Hospital, Mumbai

${ }^{3}$ Professor and Head, Department of Neurology, Topiwala National Medical College \& BYL Nair Charitable Hospital, Mumbai

Article Info: Received 20 March 2019; Accepted 15 April. 2019

Cite this article as: Bidikar, Dr. M. P., Jagtap, Dr. G., \& Chakor, Dr. R., (2019). A STUDY TO EVALUATE THE REACTION TIME IN TENSION TYPE HEADACHE. International Journal of Medical and Biomedical Studies, 3(4).

DOI: https://doi.org/10.32553/ijmbs.v3i4.198

Address for Correspondence: Dr. Mukta P Bidikar, Assistant Professor, Department of Physiology, HBT Medical College \& Dr. R N Cooper Hospital, Mumbai

Conflict of interest: No conflict of interest.

\section{Abstract}

The study evaluated the visual and auditory reaction time (simple and choice) in tension type headache patients and healthy controls.

Materials and Methods: Study included 21 Tension type headache (TTH) patients in the age group of 21- 49 years and 21 age and sex matched controls. Simple and choice auditory and visual reaction time was recorded using Medicaid Systems RTM-604. The results were statistically analyzed and were recorded as mean \pm standard deviation and Student's unpaired t-test was applied to check the level of significance.

Results: The mean values of simple visual reaction time (VRT) and auditory reaction time (ART) were not significantly different in Tension type headache patients and controls. The visual and auditory choice reaction time was longer in TTH patients as compared to controls although not statistically significant.

Conclusion: Cognitive and psychomotor impairment has been reported in headache patients. Our findings also indicate delayed central processing in TTH patients and these findings need to be further validated in larger sample size.

Keywords: Simple and choice Reaction time; Tension Type Headache

\section{Introduction}

Simple reaction time is the minimal time needed to respond to a stimulus as fast as possible. It is a measure of sensorimotor association. Reaction time is a simple and non-invasive test for peripheral as well as central neuronal processing. Helmholtz first designed a reaction time task to measure the speed of neural transmission. ${ }^{1}$ Simple reaction times were first studied by Francis Galton in $19^{\text {th }}$ century. ${ }^{2}$ With the growth of cognitive psychology, reaction time has been used extensively to study a wide variety of mental activities in both normal and neurologically impaired populations. Three types of reaction time have been described: ${ }^{3}$ 
- Simple reaction time involves one stimulus and one response.

- Recognition reaction time which includes some stimulus that needs to be responded to and other that should not get response.

- Choice reaction time involves multiple stimulus and multiple responses.

Factors that can affect reaction time include age, sex, fatigue, fasting, personality type, exercise and intelligence of the subject. Practice can also influence simple reaction time. This maybe because with repetition motor circuits of basal ganglia allow automatic execution of motor task. $^{4-6}$

Tension type headache (TTH) is the most prevalent primary headache and impacts most of the population at some point in life. The lifetime prevalence of $\mathrm{TTH}$ is $30 \%$ to $78 \%{ }^{6}$ In the present study, auditory reaction time (ART) and visual reaction time (VRT) has been studied in patients with tension type headache. There are various studies evaluating cognitive impairment in tension type headache with conflicting results. ${ }^{7,8}$ The present study thereby aims to explore the simple and choice reaction time to visual and auditory stimuli in tension type headache patients.

\section{Material and Method:}

The study was conducted in a tertiary care teaching hospital with approval from institutional ethics committee. Study population included 21 subjects comprising diagnosed patients of tension type headache and 21 age and sex matched controls. The subjects were diagnosed as per International Headache Society Diagnostic Criteria. ${ }^{9}$

ART and VRT were done using Medicaid Systems RTM-604 (Chandigarh, India). This instrument was equipped with a sensitive quartz clock which measured up to $1 / 10$ th of a msec. The readings were taken in a quiet secluded room between 10 am to $12 \mathrm{pm}$. All subjects were right handed and used their right index finger to press the button of the apparatus. Each subject was instructed to press the switch as soon as he saw the light or heard the sound. 3 practice sessions were given for each visual or auditory reaction time before starting the recording. The following simple reaction times were noted:

- VRT measurement: The examiner pressed the "start" switch in the panel which was out of view of the subject and the subject responded by pressing the "stop" button in his end of panel.

- ART measurement: Examiner presses the start button on the panel which is out of view of the subject and subject responds by pressing "stop" button on panel when he/ she hears sound ( $1000 \mathrm{~Hz}$ 's tone) through headphone.

From the auto-display, the reaction time was noted. Three readings of each stimulus were noted after giving three practical trials and their mean was taken as the reaction time. ${ }^{10}$

For recording the choice reaction time 3 stimuli red, green and yellow were used. The colours were randomly presented to the subject. The subject responded by pressing the corresponding button in front of the light. The reaction time was noted from digital display and average of three values was taken. To record auditory choice reaction time subjects were trained to differentiate between high, medium and low frequency of sound. Similar to visual choice reaction time average of three readings was taken after practice session.

\section{RESULTS:}

21 Tension type headache (TTH) patients (mean age $32 \pm 7$ ) and 21 controls (mean age $36 \pm 8$ ) were evaluated for simple reaction time tasks. Simple Visual reaction time and auditory reaction time was measured in seconds. The statistical significance was determined by the Student's unpaired " $\mathrm{t}$ " test.

The observation was taken as significant at $p<$ 0.05. There was no significant statistical difference in simple visual and auditory reaction time in TTH patients and controls. (Table 1) 
Table 1: Simple visual reaction time in TTH patients and controls

\begin{tabular}{|c|c|c|c|c|c|}
\hline \multirow[t]{3}{*}{ AGE } & \multicolumn{2}{|l|}{ TTH } & \multicolumn{2}{|c|}{ Controls } & \multirow[b]{2}{*}{$\begin{array}{l}\mathrm{P} \\
\text { value }\end{array}$} \\
\hline & Mean & $\begin{array}{l}\text { Std. } \\
\text { Deviation }\end{array}$ & Mean & $\begin{array}{l}\text { Std. } \\
\text { Deviation }\end{array}$ & \\
\hline & 32.190 & 7.903 & 36.000 & 8.198 & 0.133 \\
\hline $\begin{array}{ll}\text { SIMPLE } & \text { VISUALREACTION } \\
\text { TIME } & \\
\text { (RED LIGHT) } & \end{array}$ & 0.277 & 0.069 & 0.282 & 0.066 & 0.808 \\
\hline $\begin{array}{l}\text { SIMPLE AUDITORY REACTION } \\
\text { TIME } \\
\text { (HIGH FREQUENCY) }\end{array}$ & 0.274 & 0.071 & 0.279 & 0.067 & 0.791 \\
\hline
\end{tabular}

Table 2: Visual choice reaction time in TTH patients and controls.

\begin{tabular}{|c|c|c|c|c|c|c|}
\hline \multirow{2}{*}{$\begin{array}{l}\text { VISUAL } \\
\text { TIME }\end{array}$} & \multirow{2}{*}{ CHOICE REACTION } & \multicolumn{2}{|l|}{ TTH } & \multicolumn{2}{|c|}{ Controls } & \multirow[b]{2}{*}{$P$ value } \\
\hline & & Mean & $\begin{array}{l}\text { Std. } \\
\text { Deviation }\end{array}$ & Mean & $\begin{array}{l}\text { Std. } \\
\text { Deviation }\end{array}$ & \\
\hline Red & & 0.569 & 0.169 & 0.499 & 0.147 & 0.159 \\
\hline Green & & 0.700 & 0.172 & 0.633 & 0.150 & 0.188 \\
\hline Yellow & & 0.733 & 0.173 & 0.658 & 0.133 & 0.124 \\
\hline
\end{tabular}

The mean average choice reaction time in TTH patients for red light $(0.569 \pm 0.169)$; green light $(0.700$ $\pm 0.172)$; yellow light $(0.733 \pm 0.173)$ was longer when compared to controls although not statistically significant. The mean average visual choice reaction time values in controls were as follows : for red light $(0.499 \pm 0.147)$; green light $(0.633 \pm 0.150)$; yellow light $(0.658 \pm 0.133)$. (Table 2 ; Figure 1$)$.

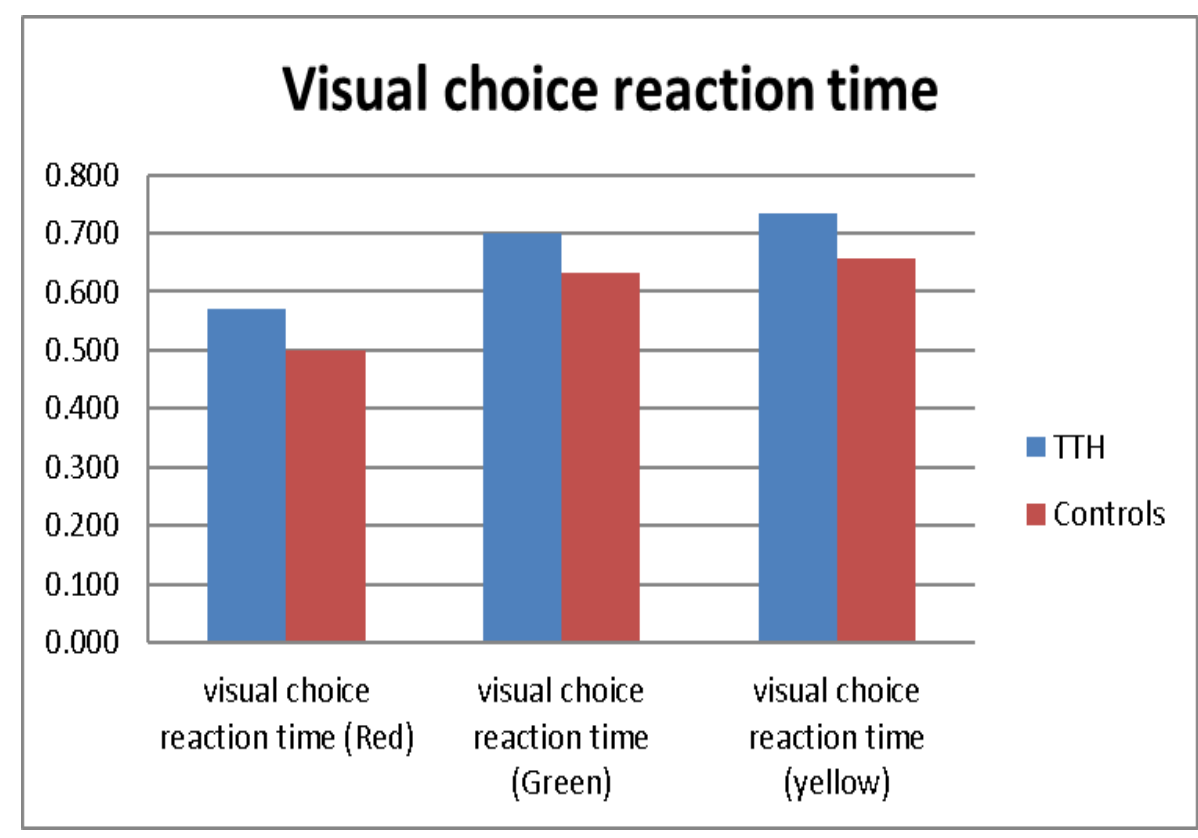

Figure 1: Visual choice reaction time in TTH patients and controls. 
Table 3: Auditory choice reaction time in TTH patients and controls.

\begin{tabular}{|c|c|c|c|c|c|}
\hline \multirow[b]{2}{*}{ AUDITORY CHOICE REACTION TIME } & \multicolumn{2}{|l|}{ TTH } & \multicolumn{2}{|c|}{ Controls } & \multirow[b]{2}{*}{$\begin{array}{l}P \\
\text { value }\end{array}$} \\
\hline & Mean & $\begin{array}{l}\text { Std. } \\
\text { Deviation }\end{array}$ & Mean & $\begin{array}{l}\text { Std. } \\
\text { Deviation }\end{array}$ & \\
\hline High frequency & 0.601 & 0.156 & 0.519 & 0.149 & 0.087 \\
\hline Medium frequency & 0.715 & 0.133 & 0.632 & 0.160 & 0.076 \\
\hline Low frequency & 0.785 & 0.171 & 0.707 & 0.162 & 0.134 \\
\hline
\end{tabular}

Similar findings were also noted for auditory choice reaction time. The mean average choice reaction time in TTH patients for high frequency $(0.601 \pm 0.156)$; medium frequency $(0.715 \pm 0.133)$; low frequency $(0.785 \pm 0.171)$ was longer when compared to controls although not statistically significant. The mean average auditory choice reaction time values in controls were as follows: for high frequency $(0.519 \pm 0.149) ;$ medium frequency $(0.632 \pm 0.160)$; yellow light $(0.707 \pm 0.162)$. (Table 3; Figure 2$)$.

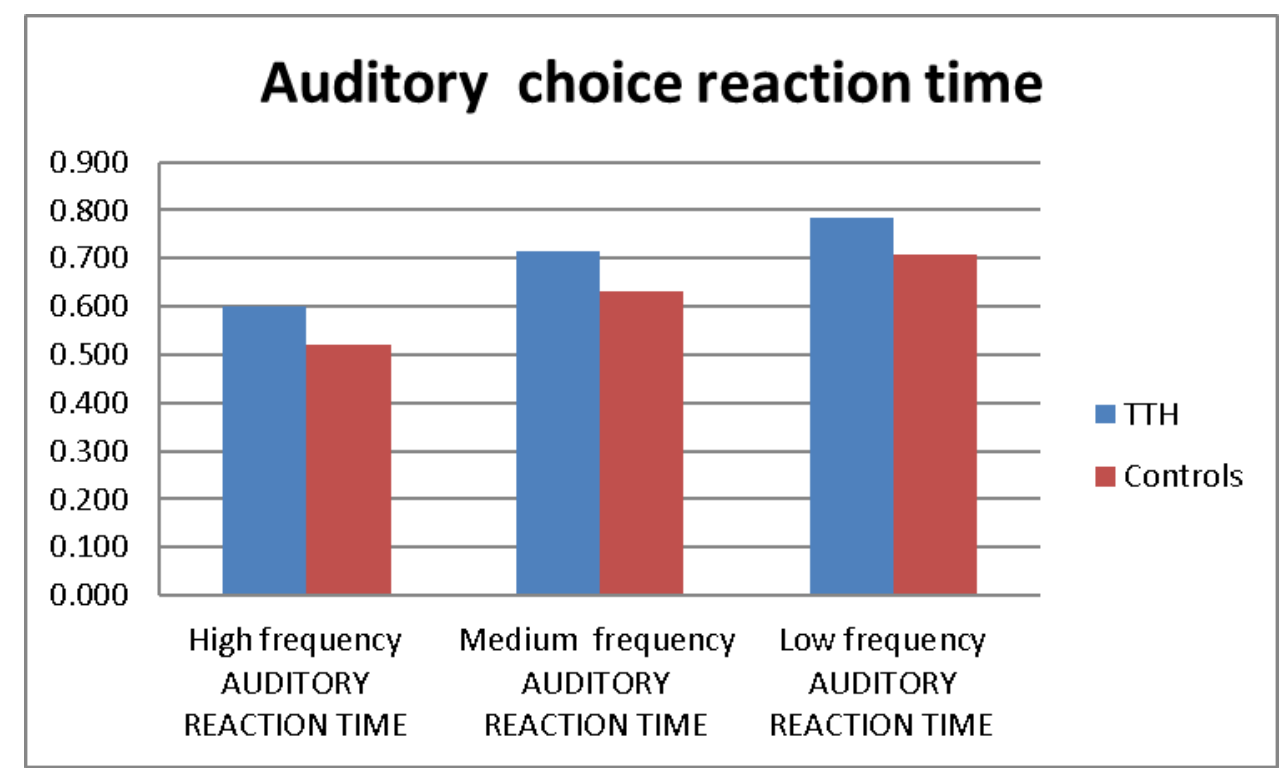

Figure 2: Auditory choice reaction time in TTH patients and controls.

\section{DISCUSSION:}

Reaction time is one of the important factors helping an animal cope with the environmental changes and for maintaining homeostasis. It is especially important when driving, playing sports, in emergency situations and in many day to day activities. ${ }^{9}$ Reaction time consists of mainly 2 components:

- Mental processing time: It is the time required for subject to perceive the stimulus, identify it and decide proper motor response.
- Movement time: It is the time required to perform the movement after selection of appropriate response.

Tension type headache has been historically ascribed to persistent contraction of scalp, neck and jaw musculature. It has been variously known as essential headache, ordinary headache, psychogenic headache and idiopathic headache. The pathogenesis of headache is not well understood and various pathophysiological factors influencing tension type headache have been suggested. Peripheral pain mechanisms are most likely to play a role in episodic tension type headache while central pain mechanisms play an 
important role in chronic tension type headache. ${ }^{11}$

In the present study we did not find significant difference in simple reaction time tasks between tension type headache patients in inter-ictal period and controls. The choice reaction time however was more prolonged in TTH patients as compared to controls although not statistically significant. This could mean that central processing was more delayed in TTH patients as compared to controls. Smith AP confirmed that having an acute TTH was associated with an increase in negative affect, poorer performance on working memory and semantic memory tasks, slower psychomotor performance, and increased distraction from irrelevant stimuli. He concluded that that an acute headache is associated with behavioural changes. ${ }^{12}$ Kuhajda $\mathrm{MC}$ et al reported decreased attention (encoding) and memory (recognition) with headache pain. ${ }^{13}$

Rasmussen BK et al found $59 \%$ of headache subjects had moderate to severe impairment in their daily activities. ${ }^{14}$ This can have implications for safe performance of daily tasks in TTH patients.

\section{CONCLUSION:}

Simple reaction time parameters for visual and auditory reaction time were not significantly affected in our study. The study needs to be done in a larger population and reaction time need to be studied during the headache period to further validate and compare the findings.

\section{References:}

1. Wichmann $T$, Buchheim A, Menning $H$, Schenk I, George C, Pokorny D. A reaction time experiment on adult attachment: The development of a measure for neurophysiological settings. Frontiers in human neuroscience. 2016 Nov 2;10:548.

2. Jensen AR, Munro E. Reaction time, movement time, and intelligence. Intelligence. 1979 Apr 1;3(2):121-6.

3. Badau D, Baydil B, Badau A. Differences among three measures of reaction time based on hand laterality in individual sports. Sports. 2018 Jun;6(2):45.

4. Taimela S. Factors affecting reaction-time testing and the interpretation of results. Perceptual and Motor skills. 1991 Dec;73 (3_suppl):1195-202.

5. Ghuntla TP, Mehta HB, Gokhale PA, Shah CJ. Influence of practice on visual reaction time. Journal of Mahatma Gandhi Institute of Medical Sciences. 2014 Jul 1;19(2):119.

6. Karia RM, Ghuntla TP, Mehta HB, Gokhale $\mathrm{PA}$, Shah CJ. Effect of gender difference on visual reaction time: $A$ study on medical students of Bhavnagar region. IOSR PHR. 2012;2:452-4.

7. Bendtsen L, Jensen R. Tension-type headache. Neurologic Clinics. 2009 May 1;27(2):525-35.

8. Waldie $K E$, Welch D. Cognitive function in tension-type headache. Current pain and headache reports. 2007 Dec 1;11(6):454-60.

9. Yang FC, Lin TY, Chen HJ, Lee JT, Lin CC, Kao $\mathrm{CH}$. Increased risk of dementia in patients with tension-type headache: a nationwide retrospective population-based cohort study. PloS one. 2016 Jun 7;11(6):e0156097.

10. Headache Classification Committee of the International Headache Society (IHS). The international classification of headache disorders, (beta version). Cephalalgia. 2013 Jul;33(9):629-808.

11. Das S, Gandhi A, Mondal S. The effect of premenstrual stress on the audiovisual reaction time and the audiogram. Ind J Physiol Pharmacol 1997; 41: 67-70.

12. Kaniecki RG. Tension-type headache. In Headache and Migraine Biology and Management 2015 Jan 1 (pp. 149-160). Academic Press.

13. Smith AP. Acute tension-type headaches are associated with impaired cognitive function and more negative mood. Frontiers in neurology. 2016 Mar 29;7:42.

14. Kuhajda $M C$, Thorn $B E$, Klinger MR, Rubin NJ. The effect of headache pain on attention (encoding) and memory (recognition) Pain 
Dr. Mukta P Bidikar et al, International Journal of Medical and Biomedical Studies (IJMBS)

(2002) 97:213-21.10. 1016/S0304-3959(01) 00488-2.

15. Rasmussen BK, Jensen R, Olesen J. Impact of headache on sickness absence and utilisation of medical services: a Danish population study. J Epidemiol Community Health (1992) 42:443-6.10.1136/jech.46.4. 443 . 